Pacific Journal of Mathematics

ON BERGMAN OPERATORS FOR PARTIAL DIFFERENTIAL
EQUATIONS IN TWO VARIABLES 


\title{
ON BERGMAN OPERATORS FOR PARTIAL DIFFERENTIAL EQUATIONS IN TWO VARIABLES
}

\section{ERWIN KREYSZIG}

\begin{abstract}
Bergman operators are linear integral operators that map complex analytic functions into solutions of linear partial differential equations with analytic coefficients. In this way methods and results of complex analysis can be used for characterizing general properties of classes of those solutions. For example, this approach yields theorems about the location and type of singularities, the growth, and the coefficient problem for series developments of solutions.

A partial differential equation being given, there exist various types of Bergman operators, and for that purpose it is essential to select an operator whose generating function is as simple as possible. The present paper considers differential equations in two independent variables, introduces a class of Bergman operators satisfying that requirement, and determines the corresponding class of differential equations in an explicit fashion. In fact, necessary and sufficient conditions are obtained in order that the solutions of a partial differential equation can be obtained by means of a Bergman operator of that class. It is also shown that the set of these equations includes several equations of practical importance.
\end{abstract}

2. Bergman operators of class $P_{0}$. We consider partial differential equations of the form

$$
\Delta \psi+\alpha(x, y) \psi_{x}+\beta(x, y) \psi_{y}+\gamma(x, y) \psi=0
$$

assuming that $\alpha, \beta$ and $\gamma$ are real analytic functions in some neighborhood of the origin. Setting $z_{1}=x+i X, z_{2}=y+i Y$, we may continue the coefficients to complex values of the variables. We now introduce the variables

$$
z=z_{1}+i z_{2} \text { and } z^{*}=z_{1}-i z_{2} \text {. }
$$

(Note that $z^{*}=\bar{z}$ if $z_{1}$ and $z_{2}$ are real.) Transforming the given equation and eliminating one of the two first partial derivatives, we obtain

$$
L u:=u_{z z^{*}}+b\left(z, z^{*}\right) u_{z^{*}}+c\left(z, z^{*}\right) u=0 .
$$

A Bergman operator $B$ corresponding to (2.1) may be defined by means of 


$$
(B f)\left(z, z^{*}\right):=\int_{-1}^{1} g\left(z, z^{*}, t\right) f\left(\frac{z}{2}\left(1-t^{2}\right)\right)\left(1-t^{2}\right)^{-1 / 2} d t
$$

( $t$ real). S. Bergman [4] has shown that if $g\left(z, z^{*}, t\right)$ is a solution of

$$
\left(1-t^{2}\right) g_{z^{*} t}-t^{-1} g_{z^{*}}+2 z t L g=0
$$

and $g_{z^{*}} / t z$ is continuous, then $u\left(z, z^{*}\right)=(B f)\left(z, z^{*}\right)$ is a solution of (2.1); here $f$ is any analytic function. $g$ is called the generating function of the operator $B$, and $f$ is called a $B$-associated function of that solution $u$.

If $L$ is such that (2.3) has a solution of the form

$$
g\left(z, z^{*}, t\right)=\sum_{\mu=0}^{m} q_{2 \mu}\left(z, z^{*}\right) t^{2 \mu}
$$

( $g_{z^{*}} / t z$ continuous), then $L$ and $B$ (with $g$ given by (2.4)) are said to be of class $P$. Note that in this polynomial (2.4) we have omitted odd powers of $t$ without loss of generality, as can be seen from (2.2).

If in (2.4), the functions $q_{2 \mu}\left(z, z^{*}\right)$ can be represented in the form

$$
q_{2 \mu}\left(z, z^{*}\right)=\lambda_{2 \mu} q\left(z, z^{*}\right)^{\mu}
$$

where $\mu=0, \cdots, m$ and $\lambda_{0}=1, \lambda_{2}, \cdots, \lambda_{2 m}$ are constants, then $L$ and $B$ (with $g$ given by $(2.4),(2.5)$ ) are said to be of class $P_{0}$.

$L$ is of class $P$ if and only if there is a natural number $m$ such that

$$
h_{2 m+2}\left(z, z^{*}\right)=0
$$

where $h_{2}\left(z, z^{*}\right)=c\left(z, z^{*}\right) q_{0}(z)$ with arbitrary analytic $q_{0}(z)$ and

$$
h_{2 \mu+2}\left(z, z^{*}\right)=\left(M_{2 \mu} h_{2 \mu}\right)\left(z, z^{*}\right) \quad(\mu=1, \cdots, m)
$$

with $M_{2 \mu}$ defined by

$$
\left(M_{2 \mu} w\right)\left(z, z^{*}\right)=\frac{2 z}{2 \mu-1}\left(w_{z}+\left(b\left(z, z^{*}\right)-\frac{\mu-1}{z}\right) w+c\left(z, z^{*}\right) \int w d z^{*}\right) .
$$

This necessary and sufficient condition for $L \in P$ was obtained in [9]. An equation (2.1) being given, it is clear that this condition may readily by used to find out whether or not $L \in P$. However, the condition does not yield an explicit characterization of the class $P$, that is, it does not give explicit expressions for the coefficients $b\left(z, z^{*}\right)$ and $c\left(z, z^{*}\right)$ of all the differential equations (2.1) with $L \in P$.

Such explicit expressions are desirable, for instance, in connection with generalizing Bauer's and Peschl's theory [1-3] of the equation 


$$
L_{0} u:=u_{z z^{*}}+\frac{\varepsilon m(m+1)}{\left(1+\varepsilon z z^{*}\right)^{2}} u=0 \quad(m=1,2, \cdots ; \varepsilon= \pm 1)
$$

to other equations. $L_{0}$ is of class $P$, and (2.6) is of importance because it is closely related to the Laplace and wave equations.

We shall now see that the class $P_{0}$, which is a subclass of the class $P$, can be characterized by necessary and sufficient conditions in an explicit fashion. Some applications will be given $\S 4$, and it will be shown that $L_{0} \in P_{0}$.

3. Explicit determination of the class $P_{0}$. For any equation (2.1) a corresponding Bergman operator $B$ [cf. (2.2)] can be obtained by determining a power series solution of (2.3), as Bergman [4] has shown. Clearly, if we choose a particular class of generating functions $g\left(z, z^{*}, t\right)$, such as (2.4), we impose certain restrictions on the coefficients $b\left(z, z^{*}\right)$ and $c\left(z, z^{*}\right)$ in (2.1), and it is of interest to find corresponding conditions for $b$ and $c$. A first class of Bergman operators for which this problem was solved is the class of exponential operators, which are suitable for the Helmholtz equation and other equations and have the property that the theory of linear ordinary differential equations can be applied in the study of solutions of partial differential equations represented by means of those operators; cf. [7] and [8]. We shall now obtain a complete solution of that problem for the class $P_{0}$.

Theorem 1. In (2.1), $L \in P_{0}$ if and only if (a) or (b) holds:

(a) There is a function $p\left(z, z^{*}\right)$ such that $b$ and $c$ in (2.1) can be represented in the form

$$
b\left(z, z^{*}\right)=\lambda p-\frac{p_{z z^{*}}}{p_{z^{*}}}, c\left(z, z^{*}\right)=-\lambda p_{z^{*}} \quad(\lambda \text { any constant }) .
$$

(b) There is an integer $m>1$ and a function $\sigma\left(z^{*}\right)$ such that $b$ and $c$ in (2.1) can be represented in the form

$$
b\left(z, z^{*}\right)=\frac{\kappa}{k z+\sigma\left(z^{*}\right)} \quad(\kappa, k \text { any constants })
$$

and

$$
c\left(z, z^{*}\right)=-m((m+1) k-\kappa) \frac{\sigma^{\prime}\left(z^{*}\right)}{\left(k z+\sigma\left(z^{*}\right)\right)^{2}} \cdot
$$

In Case (a), $m=1$ in (2.4), $\lambda_{2}=2 \lambda$ and $q\left(z, z^{*}\right)=z p\left(z, z^{*}\right)$ in (2.5). In Case (b), 


$$
\lambda_{2 \mu}=\frac{(-4)^{\mu} m !}{(m-\mu) !(2 \mu) !} \prod_{\nu=1}^{\mu}((m+\nu) k-\kappa) \quad(\mu=1, \cdots, m)
$$

and

$$
q\left(z, z^{*}\right)=\frac{z}{k z+\sigma\left(z^{*}\right)}
$$

Proof. If we substitute (2.4) in (2.3) and equate to zero the coefficient of each occurring power of $t$, we obtain a system of $m+2$ partial differential equations involving $b\left(z, z^{*}\right), c\left(z, z^{*}\right)$, and the coefficients $q_{0}\left(z, z^{*}\right), \cdots, q_{2 m}\left(z, z^{*}\right)$ and their first and second partial derivatives. Let $\langle n\rangle$ denote the equation corresponding to $t^{n}$. Then the system is

$$
\begin{array}{ll}
\langle-1\rangle & q_{0 z^{*}}=0 \\
\langle 1\rangle & q_{2 z^{*}}+2 z c q_{0}=0 \\
\langle 2 \mu-1\rangle & (2 \mu-1) q_{2 \mu z^{*}}+2 z L q_{2 \mu-2}-2(\mu-1) q_{2 \mu-2, z^{*}}=0 \\
& \quad(\mu=2, \cdots, m) \\
\langle 2 m+1\rangle & z L q_{2 m}-m q_{2 m z^{*}}=0
\end{array}
$$

(where $\langle 2 \mu-1\rangle$ must be ignored if $m=1$ ). Setting

$$
q_{2 \mu}\left(z, z^{*}\right)=z^{\mu} p_{2 \mu}\left(z, z^{*}\right),
$$

we see that the $p_{2 \mu}\left(z, z^{*}\right)$ satisfy the simpler system
(a) $p_{0 z^{*}}=0$
(b) $p_{2 z^{*}}+2 c p_{0}=0$
(c) $(2 \mu-1) p_{2 \mu z^{*}}+2 L p_{2 \mu-2}=0$
$(\mu=2, \cdots, m)$
(d) $L p_{2 m}=0$

(where (3.5c) must be ignored if $m=1$ ). We set $q\left(z, z^{*}\right)=z p\left(z, z^{*}\right)$. Then (2.5) and (3.4) imply

$$
p_{2 \mu}\left(z, z^{*}\right)=\lambda_{2 \mu} p\left(z, z^{*}\right)^{\mu} \quad(\mu=0, \cdots, m) .
$$

We have $\lambda_{0}=1$, thus $q_{0}=1$, and $(3.5 \mathrm{~b})$ now gives

$$
c\left(z, z^{*}\right)=-\frac{\lambda_{2}}{2} p_{z^{*}}
$$

Let $m=1$. Then the only other equation to be considered is $(3.5 \mathrm{~d})$ with $m=1$, and because of (3.6) and (3.7) it takes the form

$$
p_{z z^{*}}+b p_{z^{*}}-\frac{\lambda_{2}}{2} p_{z^{*}} p=0 \text {. }
$$

From this and (3.7) we obtain (3.1) where $\lambda=\lambda_{2} / 2$.

Let $m>1$. Then (3.5c) with $\mu=2$ and (3.7) give 


$$
b\left(z, z^{*}\right)=\lambda p-\frac{p_{z z^{*}}}{p_{z^{*}}} \quad \text { where } \quad \lambda=\frac{\lambda_{2}}{2}-\frac{3 \lambda_{4}}{\lambda_{2}} .
$$

Substituting this and (3.7) in (3.5c) with any $\mu, 3 \leqq \mu \leqq m$, and simplifying the resulting equation, we arrive at

$$
p_{z}=-k p^{2}
$$

where

$$
k=\frac{\lambda}{\mu-2}+\frac{1}{(\mu-1)(\mu-2)}\left\{\frac{(2 \mu-1) \mu \lambda_{2 \mu}}{2 \lambda_{2 \mu-2}}-\frac{\lambda_{2}}{2}\right\} .
$$

By integrating we have

$$
p\left(z, z^{*}\right)=\frac{1}{k z+\sigma\left(z^{*}\right)} \quad\left(\sigma\left(z^{*}\right) \text { analytic }\right)
$$

and obtain (3.3b). Furthermore, we now see that

$$
b\left(z, z^{*}\right)=(\lambda+2 k) p\left(z, z^{*}\right)
$$

and, setting $\lambda+2 k=\kappa$, we obtain (3.2a). Substituting (3.2a), (3.6)(3.8) in (3.5d), we see that a factor $\lambda_{2 m} p^{m} p_{z^{*}}$ drops out and we are left with an equation for $\lambda_{2}$. The solution is

$$
\lambda_{2}=-2 m((m+1) k-\kappa) .
$$

Form (3.9) and (3.11) it follows that

$$
\lambda_{2 \mu}=-\frac{2(m-\mu+1)}{\mu(2 \mu-1)}((m+\mu) k-\kappa) \lambda_{2 \ell-2} \quad(\mu=1, \cdots, m) .
$$

The solution is (3.3a). Finally, from $q\left(z, z^{*}\right)=z p\left(z, z^{*}\right),(3.10)$, and (3.11) we obtain (3.2b). This proves that $L \in P_{0}$ implies $(3.1)-(3.3)$. Conversely, starting from (3.1)-(3.3) we obtain $L \in P_{0}$, and the proof is complete.

4. Some applications. We first note that if $L$ is such that in (3.2b), $\kappa / k \neq m+n$ ( $n$ any natural number), then (3.3a) may be written

$$
\lambda_{2 \ell^{\prime}}=\frac{(-4 k)^{\mu} m ! \Gamma(m+\mu+1-\kappa / k)}{(m-\mu) !(2 \mu) ! \Gamma(m+1-\kappa / k)} .
$$

Taking $k=0$, we see from Theorem 1 that $L_{1}$ in the special Delassus equation

$$
L_{1} u:=u_{z z^{*}}+\frac{\kappa}{\sigma\left(z^{*}\right)} u_{z^{*}}+\frac{m \kappa \sigma^{\prime}\left(z^{*}\right)}{\sigma\left(z^{*}\right)^{2}} u=0
$$


is of class $P_{0}$ and a corresponding Bergman operator has the simple generating function

$$
g_{1}\left(z, z^{*}, t\right)=m ! \sum_{\mu=0}^{m} \frac{(4 \kappa)^{\mu}}{(m-\mu) !(2 \mu) !}\left(\frac{z t^{2}}{\sigma\left(z^{*}\right)}\right)^{\mu} .
$$

If in case (b) of Theorem 1 we require $q$ to be of the form $q\left(z, z^{*}\right)=\widetilde{q}(r)$, where $r=z z^{*}$, then $\sigma\left(z^{*}\right)=1 / z^{*}$ and the only equation with $L \in P_{0}$ satisfying that condition is

$$
u_{z z^{*}}+\kappa z^{*} \omega u_{z^{*}}+\eta \omega^{2} u=0, \quad \omega=\left(k z z^{*}+1\right)^{-1}
$$

where $\eta=m(m+1) k-\kappa$. This generalizes a theorem for the equation (2.6), recently obtained by W. Watzlawek [12] by entirely different methods.

Taking $\kappa=0$, we obtain from Theorem 1 the following theorem, which generalizes the main result in [10].

THeOREM 2. The operator $L_{2}$ defined by

$$
L_{2} u:=u_{z z^{*}}+c\left(z, z^{*}\right) u=0
$$

is of class $P_{0}$ if and only if (a) or (b) holds:

(a) $c\left(z, z^{*}\right)$ can be represented in the form $c\left(z, z^{*}\right)=\lambda p_{z^{*}}$ where $\lambda$ is any constant and $p\left(z, z^{*}\right)$ satisfies the differential equation

$$
p_{z}-\frac{\lambda}{2} p^{2}=\phi(z) \quad(\phi(z) \text { arbitrary }) .
$$

(b) There is an integer $m>1$ and a function $\sigma\left(z^{*}\right)$ such that

$$
c\left(z, z^{*}\right)=-m(m+1) k \frac{\sigma^{\prime}\left(z^{*}\right)}{\left(k z+\sigma\left(z^{*}\right)\right)^{2}} .
$$

In case (a), $m=1$ in (2.4), $\lambda_{2}=2 \lambda$ and $q\left(z, z^{*}\right)=z p\left(z, z^{*}\right)$. In Case (b),

$$
\lambda_{2 \mu^{\mu}}=(-4 k)^{\mu}\left(\begin{array}{l}
m+\mu \\
m-\mu
\end{array}\right)
$$

and $q\left(z, z^{*}\right)$ is given by $(3.3 b)$.

If $L \in P$, then (2.2) (with $g$ given by (2.4)) may be converted to a form free of integrals. In fact, $u\left(z, z^{*}\right)=(B f)\left(z, z^{*}\right)$ can then be written

$$
u\left(z, z^{*}\right)=\sum_{\mu=0}^{m} \frac{(2 \mu) !}{\mu !(4 z)^{\mu}} q_{2 \mu}\left(z, z^{*}\right) \tilde{f}^{(m-\mu)}
$$

where 


$$
\widetilde{f}(z)=\sum_{\nu=0}^{\infty} \frac{\nu ! B\left(\frac{1}{2}, \nu+\frac{1}{2}\right)}{2^{\nu}(m+\nu) !} a_{\nu} z^{m+\nu}
$$

where $a_{2}$ are the coefficients of the development

$$
f(z)=\sum_{\nu=0}^{\infty} a_{\nu} z^{\nu}
$$

This was proved in [6]. For (2.6) this representation is identical with a representation derived by $K$. W. Bauer and E. Peschl (cf. [1-3]) by means of the theory of automorphic functions and used for developing a function theory of solutions of (2.6). We mention that (2.6) was also investigated by M. Eichler [5] and I. N. Vekua [11], and a special case of (2.6) plays a role in the study of minimal surfaces by H. A. Schwarz.

Furthermore, (4.4) and results by W. Watzlawek [13] imply that the notion of fundamental systems of solutions of ordinary differential equations may be generalized to partial differential equations (2.1) with $L \in P$.

\section{REFERENCES}

1. K. W. Bauer, Über eine der Differentialgleichung $(1 \pm z \bar{z})^{2} w_{z} \pm n(n+1) \cdot w=0$ zugeordnete Funktionentheorie, Bonner Math. Schr. Nr. 28. Math. Inst. Univ. Bonn, 1965.

2. - Über die Lösungen der elliptischen Differentialgleichung $(1 \pm z \bar{z})^{2}$ $\boldsymbol{w}_{\boldsymbol{z}} \bar{z}+\lambda w=0$, J. Reine Angew. Math. 221 (1966), 48-84, 176-196.

3. K. W. Bauer and E. Peschl, Ein allgemeiner Entwicklungssatz für die Lösungen der Differentialgleichung $(1+\varepsilon z \bar{z})^{2} w_{z} \bar{z}+\varepsilon n(n+1) w=0$ in der Nähe isolierter Singularitäten, Sitz. -Ber. math. -naturw. Kl. Bayer. Akad. Wiss., München, 1965.

4. S. Bergman, Integral Operators in the Theory of Linear Partial Differential Equations, Ergebn. Math. Grenzgeb. vol. 23, 2nd rev. print. Springer, Berlin, 1969.

5. M. Eichler, Allgemeine Integration linearer partieller Differentialgleichungen von elliptischem Typ bei zwei Grundvariablen, Abh. Math. Sem. Hamburg 15 (1942), 179210.

6. M. Kracht and E. Kreyszig, Bergman-Operatoren mit Polynomen als Erzeugenden, Manuscripta Math. 1 (1969), 369-376.

7. E. Kreyszig, On a class of partial differential equations, J. Rat. Mech. Analysis 4 (1955) 907-923.

8. - On certain partial differential equations and their singularities, J. Rat. Mech. Analysis 5 (1956), 805-820.

9. — U Über zwei Klassen Bergmanscher Operatoren, Math. Nachr. 37 (1968), 197-202.

10. - Bergman-Operatoren der Klasse $P$ (In press).

11. I. N. Vekua, New Methods for Solving Elliptic Equations, Wiley, New York, 1967.

12. W. Watzlawek, Zur Lösungsdarstellung bei gewissen linearen partiellen Differentialgleichungen zueiter Ordnung (In press). 
13. — Über lineare partielle Differentialgleichungen zweiter Ordnung mit Fundamentalsystemen (In press).

Received November 26, 1969. 


\title{
PACIFIC JOURNAL OF MATHEMATICS
}

\author{
EDITORS
}

H. SAMELSON

Stanford University

Stanford, California 94305

C. R. Новву

University of Washington

Seattle, Washington 98105
J. DUGUNDJI

Department of Mathematics

University of Southern California

Los Angeles, California 90007

RICHARD ARENS

University of California

Los Angeles, California 90024

\section{ASSOCIATE EDITORS}

E. F. BECKENBACH

B. H. NeUManN

F. WOLF

K. YosHidA

\section{SUPPORTING INSTITUTIONS}

UNIVERSITY OF BRITISH COLUMBIA CALIFORNIA INSTITUTE OF TECHNOLOGY UNIVERSITY OF CALIFORNIA MONTANA STATE UNIVERSITY UNIVERSITY OF NEVADA NEW MEXICO STATE UNIVERSITY OREGON STATE UNIVERSITY UNIVERSITY OF OREGON OSAKA UNIVERSITY UNIVERSITY OF SOUTHERN CALIFORNIA
STANFORD UNIVERSITY UNIVERSITY OF TOKYO UNIVERSITY OF UTAH WASHINGTON STATE UNIVERSITY UNIVERSITY OF WASHINGTON

AMERICAN MATHEMATICAL SOCIETY CHEVRON RESEARCH CORPORATION TRW SYSTEMS

NAVAL WEAPONS CENTER 


\section{Pacific Journal of Mathematics}

\section{Vol. 36, No. $1 \quad$ November, 1971}

Norman Larrabee Alling, Analytic and harmonic obstruction on

nonorientable Klein surfaces ............................ 1

Shimshon A. Amitsur, Embeddings in matrix rings .............. 21

William Louis Armacost, The Frobenius reciprocity theorem and essentially bounded induced representations ....................... 31

Kenneth Paul Baclawski and Kenneth Kapp, Topisms and induced

non-associative systems ............................ 45

George M. Bergman, The index of a group in a semigroup ............ 55

Simeon M. Berman, Excursions above high levels for stationary Gaussian

processes....................................... 63

Peter Southcott Bullen, A criterion for $n$-convexity .............. 81

W. Homer Carlisle, III, Residual finiteness of finitely generated commutative

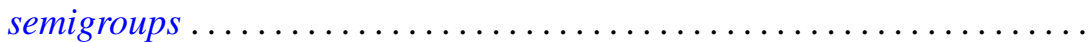

Roger Clement Crocker, On the sum of a prime and of two powers of

two ............................................ 103

David Eisenbud and Phillip Alan Griffith, The structure of serial rings . . . 109

Timothy V. Fossum, Characters and orthogonality in Frobenius

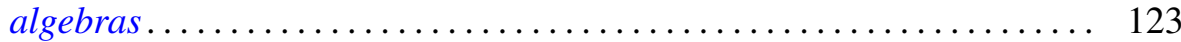

Hugh Gordon, Rings of functions determined by zero-sets . .......... 133

William Ray Hare, Jr. and John Willis Kenelly, Characterizations of Radon partitions...

Philip Hartman, On third order, nonlinear, singular boundary value

problems

David Michael Henry, Conditions for countable bases in spaces of

countable and point-countable type ..

James R. Holub, Hilbertian operators and reflexive tensor products ...

Robert P. Kaufman, Lacunary series and probability ..... . .

195

Erwin Kreyszig, On Bergman operators for partial differential equations in

two variables ................................

Chin-pi Lu, Local rings with noetherian filtrations . .

Louis Edward Narens, A nonstandard proof of the Jordan curve theorem...

S. P. Philipp, Victor Lenard Shapiro and William Hall Sills, The Abel summability of conjugate multiple Fourier-Stieltjes integrals. .

Joseph Earl Valentine and Stanley G. Wayment, Wilson angles in linear normed spaces

Hoyt D. Warner, Finite primes in simple algebras ...

Horst Günter Zimmer, An elementary proof of the Riemann hypothesis for an elliptic curve over a finite field... 